\title{
CARACTERIZACIÓN MORFOLÓGICA DE CHÍA (Salvia hispanica)
}

\author{
MORPHOLOGICAL CHARACTERIZATION OF CHÍA (Salvia hispanica)
}

\author{
José Alfonso Hernández Gómez ${ }^{1^{*}}$ y Salvador Miranda Colín ${ }^{2}$
}

\begin{abstract}
${ }^{1}$ Departamento de Fitotecnia, Universidad Autónoma Chapingo. Km. 38.5 Carretera México-Texcoco. 56230, Chapingo, Estado de México. Tel. y Fax: 01 (595) 952-1642. ${ }^{2}$ Postgrado en Recursos Genéticos y Productividad, Colegio de Postgraduados. Km. 36.5 Carretera México-Texcoco. 56230, Monteci1lo, Texcoco, Estado de México. Tel. y Fax: 01 (595) 952-0200.

* Autor para correspondencia (alhdezg2@hotmail.com)
\end{abstract}

\section{RESUMEN}

La diversidad genética en torno a la chía (Salvia hispanica L.) se ha reducido; por tanto, se requiere valorar la diversidad actual de esta especie nativa de Mesoamérica para planificar mejor su conservación y aprovechamiento. En este estudio, se evaluó la variación morfológica de 22 muestras de tipo silvestre y cultivado de $S$. hispanica. En 1999 se estableció un ensayo en un diseño de bloques completos al azar con dos plantas por bolsa y cuatro repeticiones, en condiciones de invernadero, en Chapingo, México. Se registraron 23 características morfológicas de hoja, tallo, inflorescencia, flor y semilla. Los análisis de componentes principales y de agrupamiento permitieron establecer seis grupos principales de las poblaciones de chía que se asociaron por similitudes en el ancho y largo de la corola, el ancho del cáliz, la dehiscencia de semilla, el diámetro del tallo, los días a inicio de la floración y el número de ramas. Los grupos de chía cultivada procedentes de Jalisco, Puebla y América Central, en comparación con el grupo de chía silvestre, desarrollaron la corola más ancha, más grande y expuesta; la inflorescencia más larga, más ancha y compacta; la semilla de mayor peso e indehiscente. En cambio, el grupo de chía cultivada colectada en Guerrero mostró el tamaño de la corola, el peso de la semilla y la dehiscencia de semilla similares al grupo de chía silvestre.

Palabras clave: Salvia hispanica, germoplasma, Mesoamérica.

\section{SUMMARY}

The genetic variability of chia (Salvia hispanica $L$.) has been decreasing. Thus, it is important to valorize the present diversity of Mesoamerican native chia species for a better conservation and use planning. In this study, the morphological variation of 22 cultivated and wild $S$. hispanica accessions was evaluated in 1999. Two plants of each chia accession were transplanted in randomized blocks design with four replications, under greenhouse conditions at Chapingo, México. Twenty three morphological characters of leaf, stem, inflorescence, flower and seed were recorded. Principal components and cluster analyses indicated six groups of chia accessions which were associated considering the similarity to width and length of corolla, width of calyx, seed scatter habit, stem thickness, time of flowering and branching. The cultivated chia groups from Jalisco, Puebla and Central America developed bigger, wider and more exposed corollas, longer, wider and compact inflorescences, indehiscent seeds and heavier seeds than wild chia group. On the other hand, the size of corolla, weight of seed and shatter habit of the cultivated group from Guerrero were similar to the wild chia group.
Index words: Salvia hispanica, germplasm, Mesoamerica.

\section{INTRODUCCIÓN}

"Chía" o "Chan" es un vocablo náhuatl que agrupa varias especies botánicas de los géneros Salvia, Hyptis, Amaranthus y Chenopodium; su cultivo y utilización fueron considerados por Kirchhoff (1960) como un elemento esencial de la cultura mesoamericana. Debido a que su denominación es en lengua indígena y a que existen descripciones precisas de sus formas de uso, es probable que el conocimiento y la domesticación de estas plantas se remonte a una etapa previa a la época prehispánica (Gillet, 1981).

Salvia hispanica L. es una especie cultivada de ese grupo. En la época prehispánica fue una planta importante y sus semillas, su harina o su aceite fueron apreciados por sus usos medicinales, alimenticios, artísticos y religiosos (Cahill, 2003). Actualmente, su semilla entera se usa en la preparación de una bebida nutritiva y refrescante; con el aceite extraído de sus cotiledones se elaboran lacas artesanales. S. hispanica es originaria de Mesoamérica y su mayor diversidad genética se presenta en la vertiente del Océano Pacífico (Miranda, 1978; Cahill, 2004). Se encuentra en áreas de bosque de encino o de pinoencino y se distribuye en ambientes semicálidos y templados del Eje Neovolcánico Transversal, de las Sierras Madre Occidental, del Sur y de Chiapas, en altitudes entre 1400 y $2200 \mathrm{~m}$. Al considerar su extensa área de distribución, su sistema de polinización altamente autógamo asociado con sus flores diminutas y homostílicas (Haque y Ghoshal, 1981) y la topografía accidentada de las montañas que da origen a un aislamiento geográfico de las áreas donde crece (Ramamoorthy y Elliot, 1998), es probable que exista una amplia diversificación entre poblaciones naturales de $S$. hispanica. Sin embargo, sólo se han 
descrito dos ideotipos: $S$. hispanica var. chionocalyx Fernald, con localidad tipo en Uruapan, Michoacán, y S. hispanica var. intosa Fernald, cuya localidad tipo es Buena Vista, Departamento de Sta. Rosa, Guatemala (Fernald, 1907).

Las especies cultivadas de mayor importancia en la dieta del nativo mesoamericano presentaban amplia adaptación geográfica, de tal forma que prácticamente en todas las regiones el hombre podía producir sus propios alimentos. Según Rojas (1985), existía una amplia diversidad ecológica en donde se sembraban maíz (Zea mays), frijol (Phaseolus spp.), huautli (Amaranthus leucocarpus), calabaza (Cucurbita spp.), chía (Salvia hispanica) y chile (Capsicum annuum). A pesar de su enorme importancia y diversidad de usos en la época prehispánica, la superficie cultivada y la tradición tecnológica y cultural de $S$. hispanica fue reduciéndose rápidamente a partir de la época colonial (Rojas, 1983). En 1777, la chía todavía se sembraba en Chiepetlán, Guerrero, para utilizar su aceite en la decoración de jícaras, que son recipientes elaborados a partir del fruto de Crescentia cujete (Hurtado, 1946); en Chiapas, el aceite de chía mezclado con aje (Coccus axin) se usaba en la elaboración de lacas (Miranda, 1952). En El Salvador, por lo menos hasta hace poco tiempo, la semilla de $S$. hispanica o "Chan" se empleaba para preparar refrescos o para las enfermedades del hígado (Calderón, 1941). En el periodo de 1932-1935, el cultivo de la chía en México ocupaba una superficie promedio anual de 74 ha en los Estados de Jalisco, Puebla, Michoacán, Guerrero, Oaxaca y Chiapas (Rulfo, 1937). Actualmente, se cultivada en Acatic, Jalisco, en Atzitzihuacan, Puebla, y en Olinalá, Guerrero.

La semilla de chía contiene más proteína y aceite que otros granos, por lo que sería una fuente de alimento muy atractiva para países en desarrollo (Weber et al., 1991); su aceite posee un alto contenido de ácido linolénico omega-3, esencial en la alimentación y efectivo para disminuir las afecciones cardiovasculares (FAO, 1978); los antioxidantes naturales de su aceite evitan los procesos oxidativos en alimentos (Taga et al., 1984); el mucílago de la testa de la semilla es un polisacárido útil como fibra soluble y dietética (Bushway et al., 1981); los aceites esenciales pueden usarse en la industria de saborizantes y fragancias (Ahmed et al., 1994); además, los ácidos grasos saturados y el colesterol del huevo se reducen cuando se adiciona semilla de chía a la dieta de gallinas (Ayerza y Coates, 2001). Las anteriores características han incrementado el interés comercial por Salvia hispanica, la que se ha introducido a varios países como cultivo promisorio (Coates y Ayerza, 1996) y se han industrializado diferentes productos alimenticios y medicinales preparados con semilla de chía.
La domesticación es un proceso evolutivo que opera bajo la influencia de actividades humanas. Varios autores han definido los cambios morfológicos asociados con la domesticación de algunas especies vegetales (Pernés, 1983; Harlan, 1992; Zohary y Hopf, 1994). Aunque se desconocen los orígenes de su cultivo y los procesos de su domesticación, la selección humana en $S$. hispanica ha sido una fuerza poderosa en su evolución, dada la capacidad de la planta de producir miles de semillas, combinada con su ciclo de vida anual, su sistema de polinización altamente autógamo y la alta heredabilidad de algunas características fenotípicas (Cahill y Ehdaie, 2005). Las características morfológicas y fenológicas que identifican a las variedades domesticadas de $S$. hispanica son: cálices cerrados, semilla de mayor tamaño, inflorescencias más compactas, flor más larga, presencia de dominancia apical y uniformidad en los periodos de floración y maduración (Cahill, 2005). Como en la mayoría de las plantas cultivadas, en la chía ha existido una ligera pérdida de variabilidad genética en el proceso de domesticación; en la actualidad, los esfuerzos se han dirigido a la selección de plantas domesticadas, a partir de una porción pequeña del total de la diversidad genética (Cahill, 2004).

Para reducir el deterioro genético y cultural de $S$. hispanica, se requiere un programa de rescate y valoración de esta especie nativa a fin de planificar mejor su manejo, aprovechamiento y conservación. Los objetivos de esta investigación fueron: a) identificar los patrones de variación morfológica entre poblaciones de $S$. hispanica, y b) analizar las similitudes y diferencias morfológicas dentro y entre los tipos silvestres y cultivados de $S$. hispanica.

\section{MATERIALES Y MÉTODOS}

Esta investigación se hizo en condiciones de invernadero en la Universidad Autónoma Chapingo, México, en 1999. El acopio de muestras de chía fue durante recorridos de campo, compra de semilla a productores y en mercados regionales o mediante intercambio de material con otros investigadores. Se utilizaron 22 muestras de chía, de diversa procedencia, condición y ubicación geográfica (Cuadro 1), las que están depositadas en el Banco de Germoplasma "Salvador Miranda Colín" de la Universidad Autónoma Chapingo.

Las semillas de las 22 muestras se sembraron en charolas de germinación el 26 de julio de 1999; a los 28 d de sembradas, las plántulas fueron trasplantadas a bolsas de polietileno negro de $45 \times 45 \mathrm{~cm}$, que contenían aproximadamente $18 \mathrm{~kg}$ de sustrato. Se establecieron cuatro repeticiones por muestra de chía (dos plántulas $\mathrm{x}$ bolsa $\mathrm{x}$ repetición), en un diseño de bloques completos al azar. Se aplicaron riegos periódicos y a partir de la segunda 
quincena de octubre se mantuvo a una temperatura mínima de $9{ }^{\circ} \mathrm{C}$.

Durante el desarrollo de las plantas se midió: diámetro de tallo al primer nudo $(\mathrm{cm})$; largo y ancho del limbo de la hoja ubicada en el quinto nudo a partir de la base $(\mathrm{cm})$; altura de planta $(\mathrm{cm})$, número de ramas, días a inicio de floración y periodo de floración (días). En la primera flor abierta y completamente desarrollada de cada planta se midió: largo y ancho del cáliz $(\mathrm{mm})$, longitud total del tubo de la corola, ancho del labio inferior de la corola $(\mathrm{mm})$, y largo y ancho de la bráctea basal (mm).

En la inflorescencia principal de cada planta se registró: ancho de su parte media $(\mathrm{cm})$, longitud total a partir del primer verticilo $(\mathrm{cm})$, número de verticilos y de flores en el primer verticilo. En dos muestras de cada planta se obtuvo el peso promedio de 100 semillas (g). Se identificó el tipo de cáliz de cada muestra, en los de tipo abierto, que al madurar expulsan la semilla (dehiscente), o tipo cerrado, que retendrán la semilla (indehiscente). Además, se generaron cuatro variables adicionales: relación largo/ancho del limbo, relación largo/ancho de la bráctea, distancia entre verticilos $(\mathrm{cm})$, obtenida como el cociente entre la longitud de la inflorescencia y el número de verticilos, y la exposición de corola estimada como la diferen- cia entre el largo del tubo de la corola y el largo del cáliz; valores positivos en esta variable indican que la corola sobresale del cáliz y valores negativos indican que la corola se encuentra envuelta por el cáliz.

El análisis estadístico se efectuó con el paquete SAS versión 6.12 (SAS Institute, 1998). La variable exposición de la corola fue normalizada con la transformación de la raíz cuadrada. Para precisar las variables que permitieran la mejor asociación y agrupamiento de los materiales de chía, se hizo un análisis multivariado de componentes principales, y un análisis de agrupamiento por el método Ward con los valores poblacionales promedio de las 23 variables; los grupos definidos se representaron en un dendrograma. Además, con excepción de la variable tipo de cáliz, los grupos se sometieron a un análisis de varianza con el método lineal general, y a una prueba de separación de medias con el método de Tukey $(\propto=0.05)$. Dado que el agrupamiento de las colectas separó en grupos distintos a las chías silvestres y cultivadas de diferente origen geográfico, se definieron contrastes particulares para conocer las principales diferencias y las características que permitieran una mejor diferenciación entre grupos; esos contrastes se sometieron a un análisis de varianza multivariable.

Cuadro 1. Procedencia, condición y ubicación geográfica de las 22 muestras de $S$. hispanica utilizadas en el análisis de la variación morfológica.

\begin{tabular}{|c|c|c|c|c|c|}
\hline Clave & Procedencia ${ }^{+}$ & Condición & Lat. $\mathrm{N}$ & Long. (w) & Altitud (m) \\
\hline 01 & Chihuahua. Jardín Botánico, UNAM & $\mathrm{S}$ & $27^{\circ} 03^{\prime}$ & $107^{\circ} 58^{\prime}$ & 1620 \\
\hline 02 & Chihuahua. Jardín Botánico, UNAM & $\mathrm{S}$ & $26^{\circ} 46^{\prime}$ & $106^{\circ} 45^{\prime}$ & 2200 \\
\hline 03 & Sonora, México. UC-Riverside & $\mathrm{C}$ & $28^{\circ} 13^{\prime}$ & $109^{\circ} 45^{\prime}$ & 250 \\
\hline 05 & Bosque de Pino-Encino de Tapalpa, Jalisco & S & $19^{\circ} 56^{\prime}$ & $103^{\circ} 45^{\prime}$ & 2052 \\
\hline 06 & Productores de chía de Acatic, Jalisco & $\mathrm{C}$ & $20^{\circ} 46^{\prime}$ & $102^{\circ} 54^{\prime}$ & 1680 \\
\hline 07 & Acatic. Universidad de Arizona & $\mathrm{C}$ & $32^{\circ} 13^{\prime}$ & $110^{\circ} 55^{\prime}$ & 751 \\
\hline 10 & Acatic, semilla blanca. Chapingo, México & $\mathrm{C}$ & $19^{\circ} 29^{\prime}$ & $98^{\circ} 53^{\prime}$ & 2250 \\
\hline 11 & Productores de San Mateo Cuatepec, Puebla & $\mathrm{C}$ & $18^{\circ} 48^{\prime}$ & $\begin{array}{ll}98^{\circ} & 39^{\prime}\end{array}$ & 2045 \\
\hline 12 & Mercado de Cuernavaca, Morelos & $\mathrm{C}$ & $18^{\circ} 55^{\prime}$ & $99^{\circ} 13^{\prime}$ & 1500 \\
\hline 13 & Mercado de Tepalcingo, Morelos & $\mathrm{C}$ & $18^{\circ} 54^{\prime}$ & $98^{\circ} 26^{\prime}$ & 1845 \\
\hline 14 & Mercado Sonora, D. F. & $\mathrm{C}$ & $19^{\circ} 23^{\prime}$ & $99^{\circ} 07^{\prime}$ & 2240 \\
\hline 15 & Productores de Temalacacingo, Guerrero & $\mathrm{S} / \mathrm{C}$ & $17^{\circ} 53^{\prime}$ & $98^{\circ} 40^{\prime}$ & 1480 \\
\hline 16 & Productores de Temalacacingo, Guerrero & $\mathrm{S} / \mathrm{C}$ & $17^{\circ} 53^{\prime}$ & $98^{\circ} 40^{\prime}$ & 1480 \\
\hline 21 & Mercado de San Salvador, El Salvador & $\mathrm{C}$ & $13^{\circ} 42^{\prime}$ & $89^{\circ} \quad 12$ & 690 \\
\hline 22 & Mercado de Tegucigalpa, Honduras & $\mathrm{C}$ & $14^{\circ} 05^{\prime}$ & $87^{\circ} \quad 12^{\prime}$ & 940 \\
\hline
\end{tabular}

${ }^{+}$Procedencia: Los materiales 01 y 02 fueron donados por el Dr. Robert Bye; los 03 y 04 por el Dr. Joseph P. Cahill, provenientes de la colección de Howard Gentry y el 07 por el Dr. Wayne Coates. El resto fueron obtenidos por el primer autor de esta investigación. S=Silvestre; C=Cultivada; $\mathrm{S} / \mathrm{C}=$ Cultivada con cáliz abierto al madurar (dehiscente), como en silvestres. 


\section{RESULTADOS Y DISCUSIÓN}

\section{Asociación y agrupamiento de los materiales de chía}

Del análisis de componentes principales se obtuvo que los dos primeros componentes explican $56 \%$ de la variación total $(\mathrm{CP} 1,30 \% ; \mathrm{CP} 2,26 \%)$. Cinco muestras de chía $(06,07,08,09$ y 10$)$ se ubicaron en el lado positivo y una muestra (19) en el lado negativo; y estas seis muestras se diferencian del resto con respecto a la CP1 (Figura 1). Las características y los valores propios que separan a las muestras de chía en el eje del CP1 son: ancho de corola (0.37), largo de corola (0.34) y ancho del cáliz (0.32). En la CP2 se observó una diferenciación más clara y un mejor ordenamiento, aunque varias muestras se ubicaron en la región intermedia al considerar sus valores en tipo de cáliz (0.37), diámetro del tallo (0.36), días a inicio de floración (0.33) y número de ramas (0.32). Adicionalmente, el análisis de conglomerados permitió distinguir seis grupos en las 22 muestras de chía, según su origen geográfico (Figura 2). Los seis agrupamientos resultantes son:

Grupo A. La muestras (01) y la (02) originarias de Chihuahua, la (04) de Sta. Lucía, Sinaloa, y la (05) de Tapalpa, Jalisco, se asocian en un grupo cuya procedencia se ubica al norte del país sobre la Sierra Madre Occidental. Son materiales silvestres y presentan flores pequeñas con cáliz abierto al madurar (dehiscente); son las más precoces, con tallos delgados y desarrollan el menor número de ramas.

Grupo B. Las muestras procedentes de Temalacacingo (15 y 16), la de Cuescomapa (17) y la de Chiepetlán (18), en la región de la montaña de Guerrero, se asocian en un grupo y son materiales con flores pequeñas y con el cáliz abierto al madurar (dehiscente), al igual que el grupo silvestre del norte del país, pero difieren de éste por presentar ciclos biológicos más largos, tallos más gruesos y mayor número de ramas.

Grupo C. El material colectado en San Mateo Cuatepec, Puebla (11) y en el Estado de Sonora (03) y los comprados en el mercado de Cuernavaca, Morelos (12), en la feria de Tepalcingo, Morelos (13), y en el mercado de Sonora, D. F. (14) conforman el grupo identificado como chía de Puebla, con la corola y el cáliz de tamaño intermedio que al madurar es cerrado (indehiscente); también registran valores intermedios en diámetro del tallo, número de ramas y días a inicio de floración.

Grupo D. Las muestras de origen centroamericano adquirida en los mercados de Guatemala (20), San Salvador (21) y Honduras (22) se caracterizan por tener el cáliz cerrado (indehiscente) y el tamaño de la corola y el cáliz similares al del grupo de las chías de Puebla, pero difieren de éstas en que son plantas con más días a floración, tallos más gruesos y mayor número de ramas.

Grupo E. La muestra colectada en el mercado de Oaxaca (19) tiene flores pequeñas, cuyo cáliz al madurar es cerrado (indehiscente), su ciclo biológico es el más largo, sus tallos son los más gruesos y desarrolla el mayor número de ramas.

Grupo F. La muestra comprada a productores de chía de Acatic, Jalisco (06), la colectada por el Ing. Wayne Coates (07), la adquirida en el mercado Sonora, D. F. (08) y las selecciones de semilla negra (09) y semilla blanca (10), muestran la mayor separación con respecto a los otros grupos. A este grupo se le denomina aquí como Chía de Acatic, y se caracteriza por presentar las corolas más largas y anchas, con cálices anchos y cerrados al madurar (indehiscente), e intermedios en días a floración, diámetro de tallo y número de ramas.

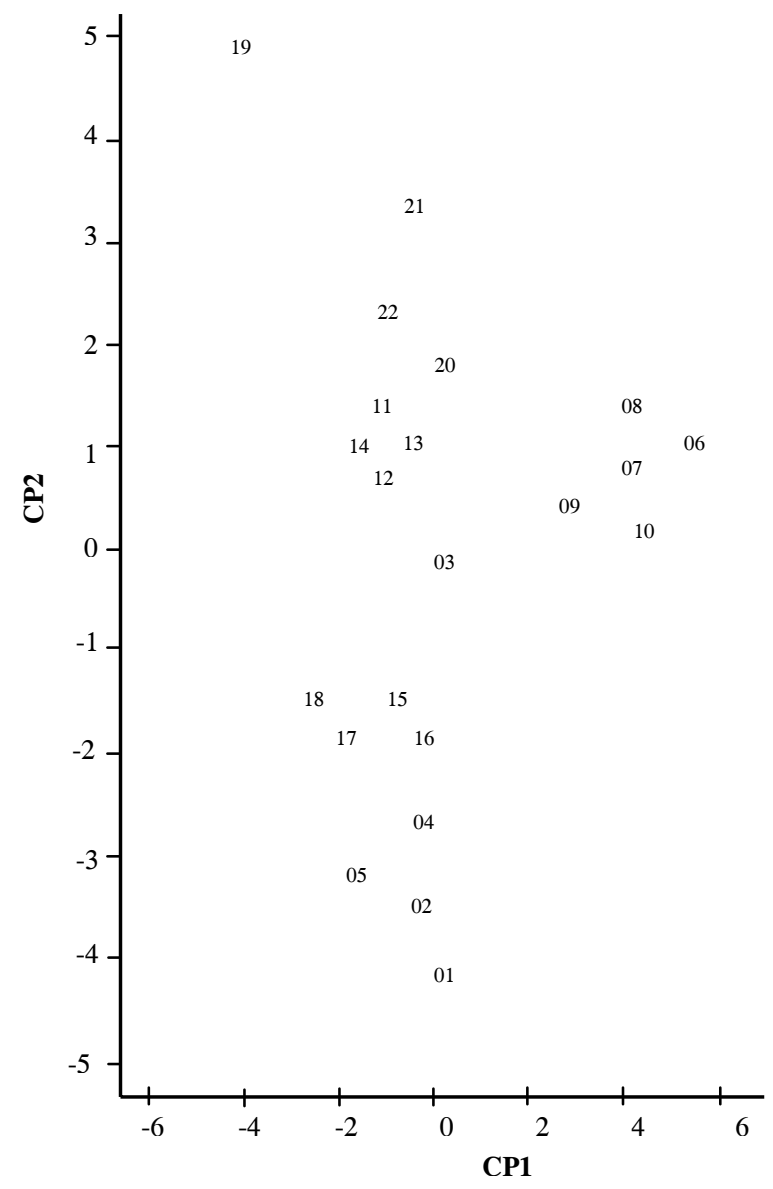

Figura 1. Primera (CP1) y segunda (CP2) componente principal del análisis de 22 materiales de chía (Salvia hispanica). En el análisis se usaron los valores medios de 23 características. La primera y segunda componente principal contabilizan 30 y $26 \%$ de la variación total, respectivamente. 


\section{Diferencias entre chía silvestre y cultivada}

Al comparar el grupo de chías silvestres del norte del país con las chías cultivadas del grupo de Acatic, Jalisco, de Puebla y de Centroamérica, se encontraron diferencias $(\mathrm{P} \leq 0.05)$ en 19 de las 23 características (Cuadro 2). $\mathrm{Al}$ considerar el valor de la $\mathrm{R}^{2}$, las variables de mayor importancia en la diferenciación de ambos grupos son: ancho de corola (0.93), días a inicio de floración (0.93), largo de corola $(0.85)$, exposición de corola $(0.84)$, distancia entre verticilos $(0.83)$, ancho del cáliz $(0.75)$, peso de semilla (0.67) y número de verticilos/inflorescencia (0.60). Las chías cultivadas se caracterizan por desarrollar flores más grandes y corolas que sobresalen del cáliz, con inflorescencias compactas debido a un mayor número de verticilos y menor distancia entre ellos; también poseen un ciclo biológico más tardío y semilla de mayor tamaño. La característica que se considera como el criterio de domesticación más importante es la indehiscencia de la semilla; la pérdida de los mecanismos naturales de dispersión de la semilla hace que las variedades domesticadas sean más dependientes del cultivo por el ser humano para sobrevivir. En las chías cultivadas de Acatic, de Puebla y las de Centroamérica, el cáliz maduro permanece cerrado, lo que evita la dispersión de semillas; en con- traste, en las chías silvestres el cáliz maduro es abierto. Estos resultados concuerdan con los de Cahill (2005) quien indicó que la domesticación en chía está definida por la presencia de cálices cerrados, de semillas más grandes, de una inflorescencia más compacta y de cierto grado de gigantismo en la flor, en la hoja y en la altura de planta.

Según Harlan et al. (1973), la presión selectiva favorece a las plantas que maduran al final de la temporada de lluvias y al inicio de la época seca; es decir, se modifica la duración del ciclo biológico. En esta investigación, los cambios observados en días al inicio de floración y en duración del periodo de floración están relacionados con las condiciones ambientales prevalecientes en la región geográfica de procedencia de las muestras. El grupo de chías silvestres es más precoz que las cultivadas, presumiblemente porque en el norte del país $\left(20^{\circ}\right.$ a $27^{\circ} \mathrm{LN}$ y de 1100 a $2200 \mathrm{msnm}$ ) el ciclo biológico se reduce por la presencia de bajas temperaturas en otoño e invierno; en contraste, las chías de Centroamérica tienen un ciclo de desarrollo más largo porque en su zona de origen $\left(13^{\circ}\right.$ a $15^{\circ} \mathrm{LN}$ y de 700 a $1514 \mathrm{msnm}$ ) las temperaturas no son limitantes y no existe una época de sequía marcada.

Cuadro 2. Valores medios, $\mathbf{R}^{2}$ y significancia de los contrastes de 23 características entre Chía silvestre vs. cultivada de Acatic, Puebla y Centroamérica (S vs. CU); Chía silvestre vs. cultivada de Guerrero (S vs. GRO); y Chía cultivada de Guerrero vs. cultivada de Acatic, Puebla y Centroamérica (GRO vs. CU).

\begin{tabular}{|c|c|c|c|c|c|c|}
\hline \multirow[b]{2}{*}{ Característica morfológica } & \multicolumn{3}{|c|}{ Medias de grupo } & \multicolumn{3}{|c|}{$\mathrm{R}^{2}$ y significancia del contraste respectivo } \\
\hline & $\mathrm{S}$ & $\mathrm{CU}$ & GRO & $\mathrm{S} v s . \mathrm{CU}$ & $\mathrm{S} v s$. GRO & GRO vs. CU \\
\hline Largo de limbo $(\mathrm{cm})$ & 11.12 & 12.71 & 12.56 & $0.26^{* *}$ & $0.33^{* *}$ & $0.16 \mathrm{~ns}$ \\
\hline Ancho de limbo $(\mathrm{cm})$ & 6.73 & 7.93 & 8.67 & $0.37^{* *}$ & $0.63^{* *}$ & $0.36^{* *}$ \\
\hline Largo/ancho de limbo & 1.66 & 1.61 & 1.44 & $0.23 \mathrm{~ns}$ & $0.38^{* *}$ & $0.64^{* *}$ \\
\hline Altura de planta $(\mathrm{cm})$ & 107.54 & 113.86 & 118.72 & $0.46^{* *}$ & $0.25^{*}$ & $0.58 \mathrm{~ns}$ \\
\hline Número de ramas & 14.87 & 18.96 & 18.87 & $0.57^{* *}$ & $0.63^{* *}$ & $0.42 \mathrm{~ns}$ \\
\hline Diámetro de tallo $(\mathrm{cm})$ & 0.65 & 0.87 & 0.82 & $0.31^{*}$ & $0.59^{* *}$ & $0.36 \mathrm{~ns}$ \\
\hline Ancho de cáliz (mm) & 2.28 & 2.60 & 2.43 & $0.75^{* *}$ & $0.13 \mathrm{~ns}$ & $0.71^{* *}$ \\
\hline Largo de cáliz (mm) & 8.06 & 8.04 & 8.31 & $0.05 \mathrm{~ns}$ & $0.28 \mathrm{~ns}$ & $0.35^{*}$ \\
\hline Largo de corola (mm) & 8.50 & 9.58 & 8.34 & $0.85^{* *}$ & $0.15 \mathrm{~ns}$ & $0.82^{* *}$ \\
\hline Ancho de corola (mm) & 4.43 & 5.51 & 4.27 & $0.93^{* *}$ & $0.11 \mathrm{~ns}$ & $0.93^{* *}$ \\
\hline Exposición de corola (mm) & 0.43 & 1.55 & -0.03 & $0.84^{* *}$ & $0.29^{*}$ & $0.79^{* *}$ \\
\hline Largo de bráctea $(\mathrm{mm})$ & 11.23 & 11.72 & 11.92 & $0.13 \mathrm{~ns}$ & $0.42^{* *}$ & $0.61 \mathrm{~ns}$ \\
\hline Ancho de bráctea (mm) & 5.98 & 5.80 & 6.95 & $0.37^{* *}$ & $0.51^{* *}$ & $0.63^{* *}$ \\
\hline Largo/ancho de bráctea & 1.90 & 2.06 & 1.72 & $0.51^{* *}$ & $0.22^{* *}$ & $0.61^{* *}$ \\
\hline Largo de inflorescencia (cm) & 21.55 & 16.06 & 16.34 & $0.49^{* *}$ & $0.41^{* *}$ & $0.15 \mathrm{~ns}$ \\
\hline Ancho de inflorescencia $(\mathrm{cm})$ & 1.41 & 1.39 & 1.48 & $0.60^{* *}$ & $0.63^{* *}$ & $0.64^{* *}$ \\
\hline Verticilos/inflorescencia & 18.97 & 21.44 & 20.20 & $0.60^{* *}$ & $0.30 \mathrm{~ns}$ & $0.43^{* *}$ \\
\hline Flores/verticilo & 16.50 & 17.11 & 18.25 & $0.46^{* *}$ & $0.35^{* *}$ & $0.51^{* *}$ \\
\hline Distancia entre verticilos $(\mathrm{cm})$ & 1.13 & 0.73 & 0.81 & $0.83^{* *}$ & $0.75^{* *}$ & $0.14^{* *}$ \\
\hline Inicio de floración (días) & 72.34 & 90.16 & 86.35 & $0.93^{* *}$ & $0.85^{* *}$ & $0.93^{* *}$ \\
\hline Periodo de floración (días) & 38.41 & 38.28 & 27.19 & $0.12 \mathrm{~ns}$ & $0.67^{* *}$ & $0.74^{* *}$ \\
\hline Peso de 100 semillas (g) & 0.101 & 0.122 & 0.105 & $0.67^{* *}$ & $0.08 \mathrm{~ns}$ & $0.82^{* *}$ \\
\hline Tipo de cáliz al madurar & Abierto & Cerrado & Abierto & & & \\
\hline
\end{tabular}

${ }^{*} \mathrm{P} \leq 0.05 ;{ }^{* *} \mathrm{P} \leq 0.01 ;$ ns, no diferencias significativas. 
Algunas adaptaciones de $S$. hispanica bajo domesticación se presentan en otras especies de origen mesoamericano, como: inflorescencia más compacta y retención del fruto al madurar, en huauzontle (Chenopodium nuttalliae Safford) (Wilson y Heiser, 1979); frutos más grandes y pesados, flores de mayor tamaño y precocidad, en tomate de cáscara (Physalis philadelphica) (Montes et al., 1991); y aumento en tamaño del fruto y número de semillas en chile (Capsicum annuum) (Rodríguez et al., 1993).

Las chías cultivadas de Guerrero presentan características morfológicas particulares. El análisis de varianza mostró que este grupo de chías difieren $(\mathrm{P} \leq 0.05)$ de las silvestres del norte de México, en 16 de 23 variables (Cuadro 2). Las de mayor relevancia en tal diferenciación, por la magnitud de las $\mathrm{R}^{2}$, son: días al inicio de floración (0.85), distancia entre verticilos (0.75), periodo de floración (0.67) y número de ramas y ancho de hoja (0.63). No difieren de las silvestres en tamaño de corola, peso de semilla y en el cáliz abierto al madurar. El hecho de que los materiales procedentes de Guerrero conserven el cáliz abierto, y tamaño de flor y de semilla como las silvestres, pero con un ciclo biológico más largo, inflorescencia más compacta y mayor número de ramas, permite inferir que estos materiales han tenido un proceso de domesticación incipiente. Cahill (2005) afirma que las chías "domesticadas primitivas" se mantienen en cultivo y son plantas que se parecen a los ecotipos silvestres típicos.

\section{Diferencias entre chías cultivadas}

Al comparar las chías cultivadas de Acatic con las de Puebla se detectaron diferencias $(\mathrm{P} \leq 0.05)$ en 18 de 23 caracteres; entre la chía de Acatic y la de Centroamérica hubo 15 características diferentes, y la chía de Puebla difiere sólo en 11 características con la de Centroamérica. Las principales variables que diferencian a los tres grupos de chía cultivada son: inicio de floración, largo y ancho de corola, ancho de inflorescencia y altura de planta. Las características en donde no se observaron diferencias son: peso de semilla, distancia entre verticilos y cáliz cerrado al madurar (Cuadro 3). La pérdida de la capacidad de dispersión de semilla, el incremento en tamaño de semilla y las inflorescencias con verticilos más cercanos pueden considerarse como cambios morfológicos comunes en las chías domesticadas. Pero también hay diferencias entre materiales cultivados; así las chías de Acatic desarrollan corolas más grandes y expuestas, inflorescencias más anchas y poseen un ciclo biológico más corto; las chías de Puebla son de ciclo biológico intermedio, con inflorescencias más pequeñas y angostas y con tamaño y exposición de la corola menores a las de Acatic; en cambio, las chías de Centroamérica tienen ciclo biológico más largo y plantas de mayor altura, con corolas similares a las de Puebla e inflorescencias parecidas a las de Acatic.

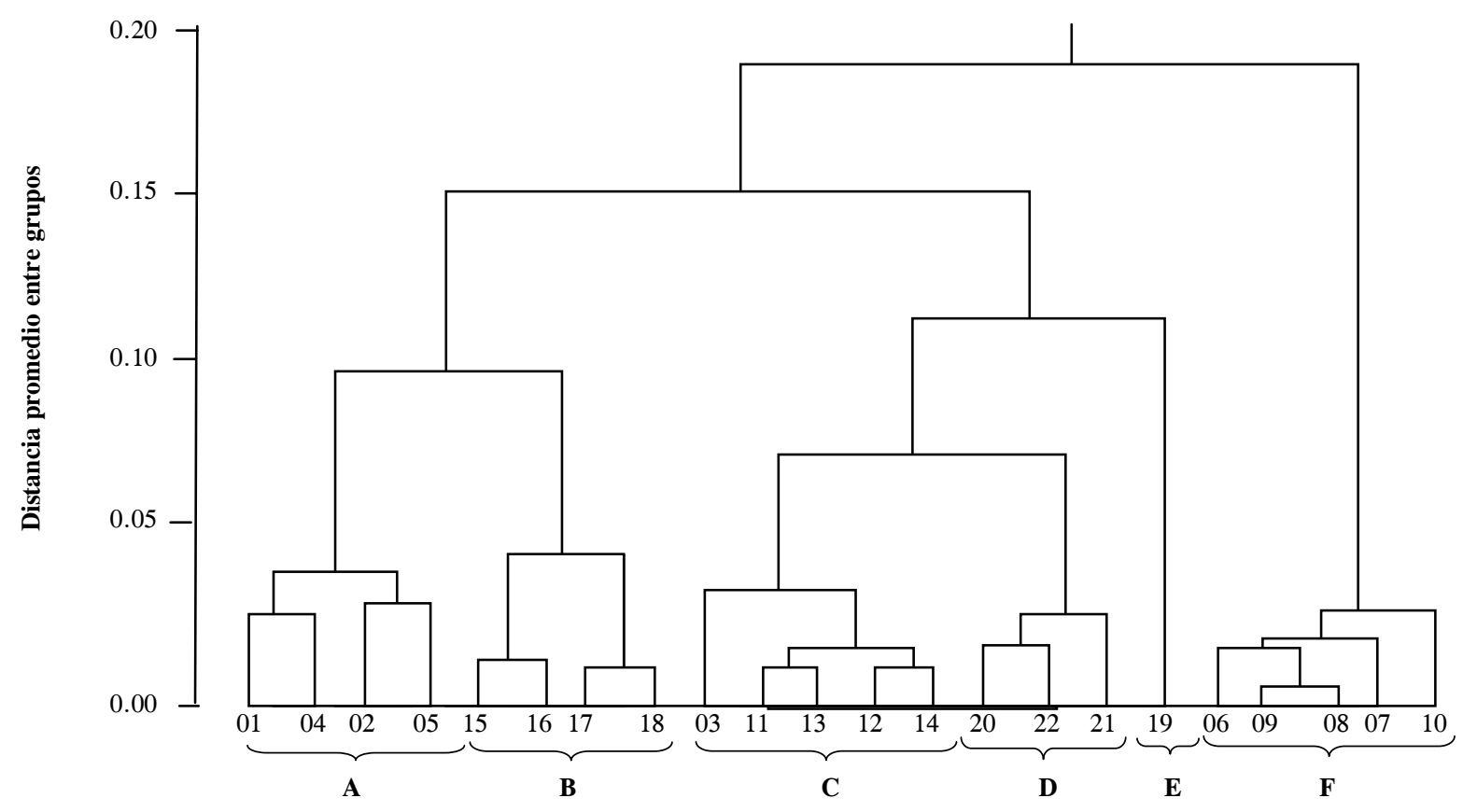

Figura 2. Dendrograma de 22 colectas de chía (Salvia hispanica). Análisis de agrupamiento de mínima varianza de Ward, basado en 23 características morfológicas. Las letras muestran los grupos formados: A, Norte de México; B, Guerrero; C, Puebla; D, Centroamérica; E, Oaxaca y F, Acatic. 
Como sucede en la mayoría de las especies cultivadas, Cahill (2004) concluyó que la diversidad genética de Salvia hispanica es mayor en las variedades silvestres que en todas las domesticadas. Sin embargo, si se considera la amplia adaptación geográfica de Salvia hispanica en condiciones de cultivo en la época prehispánica (Rojas, 1985) y el deterioro sufrido a partir del siglo XVI (Rojas, 1983), los genotipos domesticados actuales son sólo relictos de la alta diversidad genética desarrollada en Mesoamérica. En contraste, las poblaciones silvestres han sido mejor preservadas por el aislamiento geográfico que caracteriza a su amplia área de distribución natural.

Con base en los valores de $\mathrm{R}^{2}$ las chías cultivadas de Guerrero difieren de las cultivadas provenientes de Acatic, de Puebla y de Centroamérica en inicio a floración (0.93), ancho de la corola (0.93), peso de semilla (0.82), largo de corola (0.82), exposición de la corola (0.79), y en el periodo de floración (0.74), pero se diferenciaron en altura de planta, número de ramas, diámetro de tallo y longitud de inflorescencia (Cuadro 2). En los materiales cultivados, las chías de Guerrero tienen flores más pequeñas, el tubo de la corola está envuelto por el cáliz, las semillas son de menor peso, el periodo de floración es más corto y conservan el mecanismo natural de dispersión de semillas propio de las silvestres. Se conoce que la chía se cultivó en la época prehispánica en la región de la montaña de Guerrero, y que en 1777 se sembraba en Chiepetlán para extraer el aceite de su semilla y usarlo en la decoración de jícaras (Hurtado, 1946). Es probable que, posterior a esta fecha, la región haya tenido una amplia erosión genética por abandono del cultivo y que los materiales cultivados actuales provengan de poblaciones silvestres o de individuos segregantes de antiguas hibridaciones entre genotipos silvestres y domesticados. La conservación del cáliz abierto al madurar en estos materiales, podría también estar relacionada con la forma de cosechar, pues en esta región, al inicio del "amarillamiento" de las hojas las inflorescencias se cortan, se agrupan en manojos y se amarran; en esta etapa, la semilla ya alcanzó la madurez fisiológica y los cálices aún hidratados están cerrados, con lo que se evita la pérdida de semilla. Una vez que las inflorescencias están secas y los cálices abiertos, se procede a la trilla, mediante sacudidas y vibración de los manojos de inflorescencias sobre un recipiente. Según De Wet y Harlan (1975), esta forma de trilla permite seleccionar automáticamente a favor del mecanismo natural de dispersión de semillas de las plantas silvestres.

Cuadro 3. Valores medios, $\mathbf{R}^{2}$ y significancia de los contrastes de 23 características entre Chía cultivada de Acatic vs. cultivada de Puebla (A vs. P); Chía cultivada de Acatic vs. cultivada de Centroamérica (A vs. Ce); Chía cultivada de Puebla vs. cultivada de Centroamérica (P vs. Ce); y Chía de Guerrero zona cálida vs. Guerrero zona templada (Gcal vs. Gtem).

\begin{tabular}{|c|c|c|c|c|c|c|c|c|c|}
\hline \multirow{2}{*}{ Característica morfológica } & \multicolumn{5}{|c|}{ Medias de grupo } & \multicolumn{4}{|c|}{$\mathrm{R}^{2}$ y significancia del contraste respectivo } \\
\hline & A & $\mathrm{P}$ & $\mathrm{Ce}$ & Gcal & Gtem & $\begin{array}{c}\text { A } v s . \\
\mathrm{P}\end{array}$ & $\begin{array}{l}\text { A } v s . \\
\text { Ce }\end{array}$ & $\begin{array}{c}\mathrm{P} v s . \\
\mathrm{Ce}\end{array}$ & $\begin{array}{l}\text { Gcal vs. } \\
\text { Gtem }\end{array}$ \\
\hline Largo de limbo $(\mathrm{cm})$ & 12.25 & 12.24 & 13.65 & 13.06 & 12.06 & $0.04 \mathrm{~ns}$ & $0.21^{*}$ & $0.21^{*}$ & $0.68^{*}$ \\
\hline Ancho de limbo (cm) & 7.09 & 8.20 & 8.51 & 9.16 & 8.18 & $0.32^{* *}$ & $0.40^{* *}$ & $0.10 \mathrm{~ns}$ & $0.47^{*}$ \\
\hline Largo/ancho de limbo & 1.74 & 1.49 & 1.60 & 1.42 & 1.45 & $0.62^{* *}$ & $0.31^{* *}$ & $0.33^{* *}$ & $0.36 \mathrm{~ns}$ \\
\hline Altura de planta $(\mathrm{cm})$ & 99.07 & 110.01 & 132.51 & 113.12 & 124.31 & $0.38^{* *}$ & $0.77^{* *}$ & $0.67^{* *}$ & $0.63^{*}$ \\
\hline Número de ramas & 16.88 & 20.03 & 19.96 & 17.19 & 20.56 & $0.56^{* *}$ & $0.45^{* *}$ & $0.30 \mathrm{~ns}$ & $0.68^{* *}$ \\
\hline Diámetro de tallo (cm) & 0.77 & 0.82 & 1.01 & 0.77 & 0.86 & $0.22 \mathrm{~ns}$ & $0.45^{* *}$ & $0.33^{* *}$ & $0.36^{*}$ \\
\hline Ancho de cáliz (mm) & 2.91 & 2.43 & 2.47 & 2.44 & 2.42 & $0.75^{* *}$ & $0.68^{* *}$ & $0.16 \mathrm{~ns}$ & $0.56 \mathrm{~ns}$ \\
\hline Largo de cáliz (mm) & 8.18 & 7.69 & 8.24 & 8.59 & 8.03 & $0.31^{* *}$ & $0.01 \mathrm{~ns}$ & $0.45^{* *}$ & $0.49^{*}$ \\
\hline Largo de corola (mm) & 10.34 & 9.05 & 9.36 & 8.43 & 8.14 & $0.80^{* *}$ & $0.71^{* *}$ & $0.25 \mathrm{~ns}$ & $0.35 \mathrm{~ns}$ \\
\hline Ancho de corola (mm) & 7.20 & 4.70 & 4.62 & 4.25 & 4.28 & $0.93^{* *}$ & $0.92^{* *}$ & $0.15 \mathrm{~ns}$ & $0.43 \mathrm{~ns}$ \\
\hline Exposición de corola (mm) & 2.16 & 1.36 & 1.13 & -0.16 & 0.11 & $0.50^{* *}$ & $0.71^{* *}$ & $0.21 \mathrm{~ns}$ & $0.35 \mathrm{~ns}$ \\
\hline Largo de bráctea (mm) & 11.51 & 10.36 & 13.29 & 12.50 & 11.33 & $0.38^{* *}$ & $0.45^{* *}$ & $0.74^{* *}$ & $0.62 \mathrm{~ns}$ \\
\hline Ancho de bráctea (mm) & 5.01 & 6.21 & 6.18 & 7.44 & 6.46 & $0.48^{* *}$ & $0.57^{* *}$ & $0.19 \mathrm{~ns}$ & $0.80^{* *}$ \\
\hline Largo/ancho de bráctea & 2.33 & 1.68 & 2.16 & 1.68 & 1.76 & $0.61^{* *}$ & $0.09 \mathrm{~ns}$ & $0.55^{* *}$ & $0.22 \mathrm{~ns}$ \\
\hline Largo de inflorescencia $(\mathrm{cm})$ & 17.28 & 14.88 & 16.03 & 17.41 & 5.26 & $0.28^{* *}$ & $0.12 \mathrm{~ns}$ & $0.08 \mathrm{~ns}$ & $0.25 \mathrm{~ns}$ \\
\hline Ancho de inflorescencia $(\mathrm{cm})$ & 1.48 & 1.28 & 1.42 & 1.49 & 1.46 & $0.73^{* *}$ & $0.26^{*}$ & $0.74^{* *}$ & $0.05 \mathrm{~ns}$ \\
\hline Verticilos/inflorescencia & 22.75 & 18.75 & 22.83 & 21.69 & 18.75 & $0.42^{* *}$ & $0.07 \mathrm{~ns}$ & $0.44^{* *}$ & $0.37^{* *}$ \\
\hline Flores/verticilo & 19.73 & 15.23 & 16.38 & 18.50 & 18.00 & $0.60^{* *}$ & $0.48^{* *}$ & $0.14 \mathrm{~ns}$ & $0.18 \mathrm{~ns}$ \\
\hline Distancia entre verticilos $(\mathrm{cm})$ & 0.76 & 0.72 & 0.70 & 0.80 & 0.81 & $0.08 \mathrm{~ns}$ & $0.19 \mathrm{~ns}$ & $0.06 \mathrm{~ns}$ & $0.28 \mathrm{~ns}$ \\
\hline Inicio de floración (días) & 77.75 & 88.55 & 104.17 & 82.56 & 90.13 & $0.82^{* *}$ & $0.98^{* *}$ & $0.87^{* *}$ & $0.84^{* *}$ \\
\hline Periodo de floración (días) & 38.68 & 35.08 & 41.08 & 26.31 & 28.06 & $0.31^{* *}$ & $0.14 \mathrm{~ns}$ & $0.54^{* *}$ & $0.41 \mathrm{~ns}$ \\
\hline Peso de 100 semillas (g) & 0.123 & 0.122 & 0.120 & 0.116 & 0.085 & $0.02 \mathrm{~ns}$ & $0.10 \mathrm{~ns}$ & $0.20 \mathrm{~ns}$ & $0.96^{* *}$ \\
\hline Tipo de cáliz al madurar & Cerrado & Cerrado & Cerrado & Abierto & Abierto & & & & \\
\hline
\end{tabular}

${ }^{*} \mathrm{P} \leq 0.05 ;{ }^{* *} \mathrm{P} \leq 0.01 ; \mathrm{ns}$, no diferencias significativas. 
En el grupo de chías de Guerrero existen dos colectas provenientes de la región cálida de Temalacacingo $\left(17^{\circ} 53^{\circ}\right.$ LN y $1480 \mathrm{msnm}$ ) y otras dos de la región templada de Cuescomapa $\left(17^{\circ} 34^{\prime} \mathrm{LN}\right.$ y $\left.1980 \mathrm{msnm}\right)$ y Chiepetlán $\left(17^{\circ}\right.$ 43' LN y $1720 \mathrm{msnm}$ ). Cuando se compararon ambos subgrupos, el análisis estadístico mostró que su diferencia se debe a cambios en peso de semilla $\left(R^{2}=0.96\right)$, en días a inicio de floración $\left(\mathrm{R}^{2}=0.84\right)$, en número de ramas $\left(R^{2}=0.68\right)$ y en altura de planta $\left(R^{2}=0.63\right)$, pero no en forma de hoja, tamaño de flor, tamaño de inflorescencia ni periodo de floración (Cuadro 3). Es probable que las condiciones ambientales y las técnicas de manejo del cultivo particulares en cada región hayan influido en las diferencias morfológicas, de manera que en Temalacacingo, con menor altitud y diferente intensidad de cultivo, se desarrollaron materiales con ciclo biológico más corto, menor número de ramas, menor altura de planta y semillas de mayor peso, que en los materiales de la región templada. La muestra de chía de Cuescomapa produce sólo semillas con testa de color blanco. Cahill y Provance (2002) afirman que esta característica es rara en poblaciones silvestres y su expresión genética está controlada por un gene recesivo simple, lo que refuerza la idea de la intervención del hombre en el proceso evolutivo de los materiales de Guerrero.

Los resultados de esta investigación muestran que la flor, la inflorescencia, la semilla, el tipo de cáliz al madurar y el ciclo biológico, son las características morfológicas que han resultado modificadas en las chías cultivadas, con respecto a las silvestres. No obstante, existen materiales cultivados que han conservado ciertas características típicas de los materiales silvestres, como tamaño de flor, peso de semilla y tipo de cáliz, lo que indica que el proceso de diferenciación ha sido discontinuo y, por tanto, que hay un gradiente en la domesticación. La duración del ciclo biológico es la variable que representa un gran peso en todos los contrastes de diferenciación entre grupos, lo que ratifica que la diversidad de ambientes en la zona de distribución de $S$. hispanica es un importante factor en su diferenciación morfológica. Esta especie se distribuye en una amplia zona de la vertiente del Océano Pacífico, desde Centroamérica hasta el Estado de Chihuahua en México, de condiciones ambientales diversas, donde se han desarrollado numerosas culturas y se han utilizado diferentes intensidades de cultivo, por lo que es probable que el proceso de domesticación en esta especie se desarrollara en forma paulatina e independiente, de modo que los genotipos de chía se fueron adaptando a las condiciones ambientales, culturales y de cultivo propias de cada región. El transporte de material y su dispersión a nuevos ambientes, el intercambio de germoplasma y las hibridaciones constantes pudieron haber resultado en diferencia- ciones morfológicas como las que se documentan en esta investigación.

Según Cahill (2003), el deterioro genético actual de la chía está asociado con la alteración del hábitat natural o con la migración de los residentes originarios en lugares donde prosperan las poblaciones silvestres. En México, la producción de semilla de chía se enfrenta a una demanda nacional cada vez más restringida y estacional, y su aceite está siendo sustituido por el de linaza (Linum usitatissimum) en la elaboración de lacas artesanales; aunque, el interés comercial y la investigación científica sobre Salvia hispanica ha aumentado. Se requiere entonces implementar un programa de colecta de gran alcance a lo largo del área de distribución de $S$. hispanica, evaluar las nuevas poblaciones silvestres y genotipos cultivados, rescatar el conocimiento sobre las técnicas de manejo agrícola y valorar los avances científicos recientes, para sentar las bases de un programa nacional de investigación y de utilización de la especie.

\section{CONCLUSIONES}

Las características morfológicas que permiten una mejor asociación de las muestras de $S$. hispanica con su origen geográfico son: ancho y largo de corola, ancho del cáliz, tipo de cáliz, días a la floración, diámetro de tallo y número de ramas. Las estructuras morfológicas que diferencian a las muestras cultivadas de $S$. hispanica de Jalisco, de Puebla y de Centroamérica con respecto a las poblaciones silvestres, son: tamaño de flor, densidad de verticilos en la inflorescencia, peso de semilla, tipo de cáliz al madurar y la duración del ciclo biológico.

Estos tres grupos de chía cultivada son similares en tamaño de semilla, en densidad de la inflorescencia y en el cáliz cerrado al madurar, pero se diferencian en la duración del ciclo biológico, en el largo y ancho de corola, en el ancho de inflorescencia y en la altura de planta. Otro grupo de chía cultivada procedente de Guerrero muestra adaptaciones morfológicas propias de la selección bajo cultivo, como inflorescencias compactas, menor duración del periodo de floración y mayor número de ramas, pero mantiene el tamaño del cáliz y de corola, el peso de semilla y el cáliz abierto similares a las silvestres.

\section{BIBLIOGRAFÍA}

Ahmed M, I P Ting, R W Scora (1994) Leaf oil composition of Salvia hispanica L. from three geographical areas. J. Essentials Oil Res. 6:223-228.

Ayerza R, W Coates (2001) The omega-3 enriched eggs: the influence of dietary linolenic fatty acid source combination on egg production and composition. Can. J. Animal Sci. 81:355-362. 
Bushway A A, P R Belyea, R J Bushway (1981) Chia seed as source of oil, polysaccharide, and protein. J. Food Sci. 46:13491350.

Cahill J P (2003) Ethnobotany of chia, Salvia hispanica L. Econ. Bot. 57:604-618.

Cahill J P (2004) Genetic diversity among varieties of chia (Salvia hispanica L.). Gen. Res. Crop Evol. 51:773-781.

Cahill J P (2005) Human selection and domestication of chia (Salvia hispanica L.). J. Ethnobiol. 25:155-174.

Cahill J P, M C Provance (2002) Genetics of qualitative traits in domesticated Chia (Salvia hispanica L.). Heredity 93:52-55.

Cahill J P, B Ehdaie (2005) Variation and inheritance of seed mass in chia (Salvia hispanica L.). Gen. Res. Crop Evol. 52:201207.

Calderón S (1941) Flora Salvadoreña. Lista Preliminar de Plantas de El Salvador. 2a ed., corregida y aumentada. Imprenta Nacional, San Salvador, El Salvador, C. A. 442 p.

Coates W, R Ayerza (1996) Production potencial of chia in northwestwern Argentina. Indust. Crops Prod. 5:229-233.

De Wet J M J, J R Harlan (1975) Weeds and domesticates: evolution in the man-made habitat. Econ. Bot. 29:99-107.

FAO (1978) Las Grasas y Aceites en la Nutrición Humana. Informe de Expertos. Organización de las Naciones Unidas para la Agricultura y la Alimentación. Roma. 90 p.

Fernald M L (1907) Diagnoses of new spermatophytes from Mexico. Proc. Amer. Acad. Arts 43:63.

Gillet H (1981) Le chia, graine mucilagineuse mexicaine, fait son apparition en France. J. Agric. Trad. Bot. Appl. 28:183-187.

Haque M S, K K Ghoshal (1981) Floral biology and breeding system in the genus Salvia L. Proc. Indian Nat. Sci. Acad. B47:716724.

Harlan J R (1992) Crops and Man. 2nd ed. Am. Soc. Agron. Madison, Wisconsin, USA. $284 \mathrm{p}$.

Harlan J R, J M J de Wet, E G Price (1973) Comparative evolution of cereals. Evolution 27:311-325.

Hurtado de Mendoza J M (1946) La relación de Chiepetlán, Guerrero (1777). Mem. Acad. Mex. Historia 5:239-256.

Kirchhoff P (1960) Mesoamérica: Sus Límites Geográficos, Composición Étnica y Caracteres Culturales. Supl. Rev. Tlatoani (3). Escuela Nacional de Antropología e Historia. México, D. F. $15 \mathrm{p}$.

Miranda F (1952) La Vegetación de Chiapas. Primera parte. Ediciones del Gobierno del Estado. Sección autográfica. Departamento de Prensa y Turismo. Tuxtla Gutiérrez, Chiapas, México. $\mathrm{pp}: 311-312$.

Miranda C S (1978) Evolución de cultivares nativos de México. Ciencia y Desarrollo 3:130-131.

Montes H S, J R Aguirre R, E García M, F V González C (1991) Algunos efectos de la domesticación sobre la morfología del tomate (Physalis philadelphica). Agrociencia 2:7-25.

Pernés J (1983) La genética de la domesticación de los cereales. In Mundo Científico (La Recherche, en castellano, Barcelona, España) 29:964-974.

Ramamoorthy T P, M Elliot (1998) Lamiaceae de México: diversidad, distribución, endemismo y evolución. In: Diversidad Biológica de México: Orígenes y Distribución. T P Ramamoorthy, R Bye, A Lot, J Fa (eds). Primera ed. en español. Universidad Autónoma de México, México, D. F. pp:501-526.

Rodríguez M R, S Miranda C, J G Salinas G (1993) Evolución del sistema reproductivo del chile (Capsicum annuum). Agrociencia 3:121-131.

Rojas R M T (1983) La Agricultura Chinampera. Compilación Histórica UACh, Dirección de Difusión Cultural. Colección Cuadernos Universitarios, Agronomía \# 7. Chapingo, México. pp:181-203.

Rojas R M T (1985) La tecnología agrícola mesoamericana en el siglo XVI. In: Historia de la Agricultura. Época Prehispánica-Siglo XVI. Colección Biblioteca del INAH, Tomo 1. México, D. F. pp:129-231

Rulfo J M (1937) La chía. Agricultura (México) 1:28-37.

SAS Institute (1998) SAS/STAT User's Guide. Version 6.12. SAS Inst., Inc. Cary, NC.

Taga M S, E E Miller, D E Pratt (1984) Chia seeds as a source of natural lipid antioxidants. J. Am. Oil Chem. Soc. 61:928 931.

Weber C W, H S Gentry, E A Kohlhepp, P R McCrohan (1991) The nutritional and chemical evaluation of chia seeds. Ecol. Food Nutr. 26:119-125.

Wilson H D, C B Heiser (1979) The origin and evolutionary relationships of "huauzontle" (Chenopodium nuttalliae Safford), domesticated chenopod of Mexico. Amer. J. Bot. 66:198206.

Zohary D, M Hopf (1994) Domestication of Plants in the Old World. 2nd ed. Oxford University Press, New York. 279 p. 